\title{
Calcified aortic valve disease complicated with and without diabetes mellitus: the underlying pathogenesis
}

\author{
Yujia Chen ${ }^{1}$, Feng Xiao ${ }^{2, *}$, Ruxing Wang ${ }^{2, *}$ \\ ${ }^{1}$ Wuxi Clinical Medical College Affiliated to Nanjing Medical University, 214023 Wuxi, Jiangsu, China \\ ${ }^{2}$ Department of Cardiology, Wuxi People's Hospital Affiliated to Nanjing Medical University, 214023 Wuxi, Jiangsu, China \\ *Correspondence: ruxingw@sina.com (Ruxing Wang); xiaofeng@njmu.edu.cn (Feng Xiao) \\ Academic Editors: Ichiro Wakabayashi and Klaus Groschner \\ Submitted: 25 September 2021 Revised: 10 November 2021 Accepted: 22 November 2021 Published: 11 January 2022
}

\begin{abstract}
As the most prevalent valvular heart disease, calcific aortic valve disease (CAVD) is a major health problem with risk of severe morbidity and mortality in the absence of effective medical treatment beyond surgical or interventional aortic valve replacement. The pathology involved in CAVD is multifactorial, including valvular endothelial cells damage, valvular interstitial cells differentiation, extracellular matrix remodeling, inflammation, fibrosis and calcification. Various risk factors for CAVD have been reported, such as age, gender, smoking, hyperlipidemia, hypertension, obesity and bicuspid aortic valves. Recently, diabetes mellitus has also been shown to accelerate the progression of CAVD. CAVD patients complicated with diabetes mellitus may benefit from early aortic valve replacement when compared with those without diabetes mellitus. Hence, diabetes mellitus is considered as an independent risk factor for CAVD. Therefore, in-depth understanding of the pathogenesis of these two diseases and their relationship may help us find appropriate prevention and therapeutic strategies for CAVD patients complicated with diabetes mellitus.
\end{abstract}

Keywords: Calcific aortic valve disease; Diabetes mellitus; Pathogenesis; Inflammation; Fibrosis

\section{Introduction}

With the aging of the population and the increase of obesity, the prevalence of both calcific aortic valve disease (CAVD) and diabetes mellitus (DM) has been gradually increasing, and have seriously endangered the public health burden [1]. The number of patients with CAVD is expected to increase from 2.5 million in 2000 to 4.5 million in 2030 [2]. In general population, over $30 \%$ of individuals beyond the age of 65 years have echocardiography evidence of calcific aortic valve sclerosis, which is the earliest manifestation of CAVD. While calcific aortic valve stenosis affects almost $3 \%$ of adults older than 65 years of age, already $8 \%$ of adults older than 75 years of age are affected. At this stage, surgical aortic valve replacement (AVR) or transcatheter aortic valve implantation (TAVI) is the only available treatment for these patients. As a progressive disease, the pathogenesis of CAVD includes three stages. In the early stage, valvular endothelial cells (VECs) injury, lipid deposition, and inflammation constitute an initiation phase; in the late stage, valvular interstitial cells (VICs) differentiation, microcalcification, collagen and bone-matrix proteins deposition are promoted; finally, valvular osteogenesis occurs through activation of specific molecular signals $[3,4]$. Age, gender, smoking, hyperlipidemia, hypertension and obesity are all contributing factors for the high risk of CAVD. Recently, numerous studies have verified DM as an important independent risk factor for CAVD [5,6]. The prevalence of CAVD and DM is high, and DM has been shown to accelerate the progress of CAVD [7]. However, little is understood about the precise nature and underlying mechanisms of CAVD and DM [8-10]. This review aims to analyze the association between these two chronic diseases when present concurrently, and identify possible specific indicators that may be the therapeutic targets in the future.

\section{Methods}

PubMed database was searched with the following keywords: "Calcific aortic valve disease and diabetes mellitus"; "Calcific aortic valve disease" and "Diabetes mellitus". We also reviewed articles on the concepts of "Calcific aortic valve disease and inflammation" and "Calcific aortic valve disease and fibrosis". Review articles and original articles published only in English before July 2021 were included, especially those reported in the last five years.

\section{Overview of calcific aortic valve disease}

As the most common heart valvular disease in elderly patients [11], CAVD is a progressive and multifaceted process [12], that includes VECs damage, VICs differentiation, osteoblasts formation, and subsequently valve ossification, calcification, and finally aortic valve stenosis [13]. Valve calcification is an active process driven by resident VICs [14]. These cells are subjected to variety of pathological stimuli, such as inflammatory mediators, endothelial injury, low-density lipoprotein (LDL) accumulation, reactive oxygen species (ROS), and fibrosis [3]. Furthermore, VICs obtain the characteristics of osteogenesis and calcification and in turn, increase the level of calcium/phosphorus 


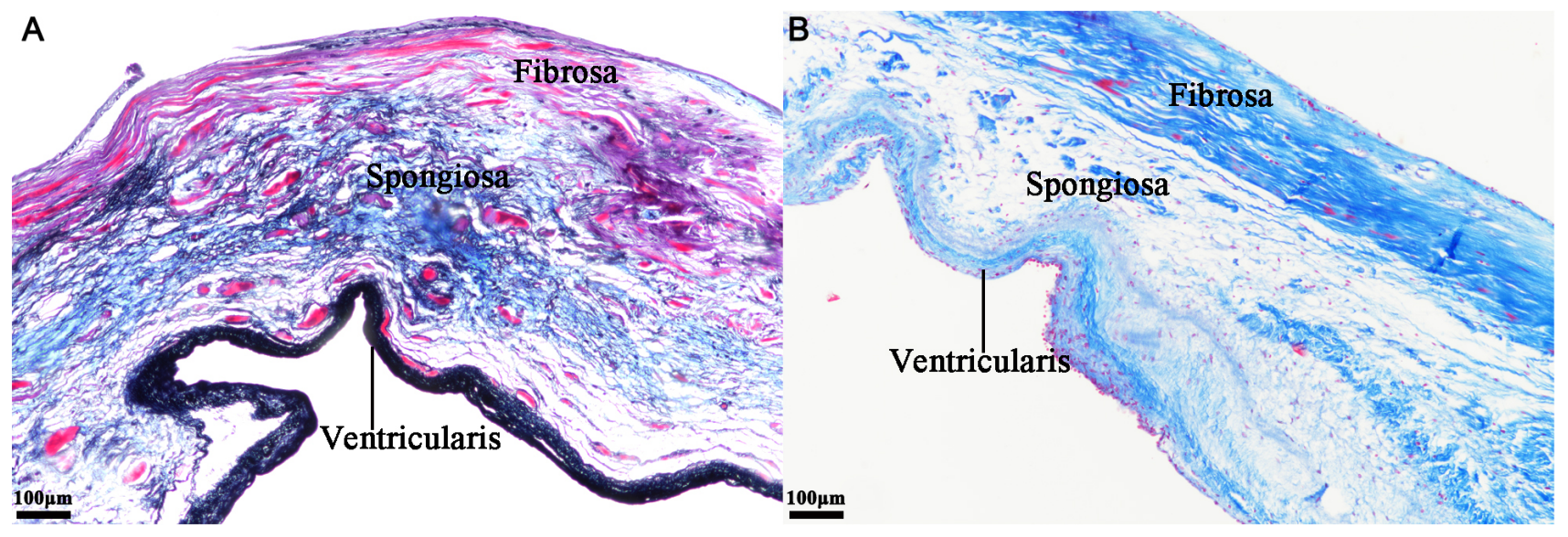

Fig. 1. The histology of aortic valve. (A) Movat's pentachrome staining of one leaflet of human calcified aortic valve exhibits valve structure. Three stratified layers are composed of collagen-rich fibrosa layer, the glycosaminoglycan-rich spongiosa layer, and the elastinrich ventricularis layer respectively. (B) Masson's trichrome staining for collagen shows the three layers of another human calcified aortic valve leaflet.

(CA/PI), modify lipids, and stretch circularly that eventually leads to valve calcification. However, the mechanism of pathogenesis of aortic valve calcification is still unclear. Therefore, without effective pharmacological therapy, symptomatic CAVD patients can only benefit from surgical AVR or TAVI. Hence, future studies on the signaling mechanisms underlying the pathogenesis of CAVD may have to identify new effective therapeutics targets for the disease.

\section{Pathophysiology of calcific aortic valve disease}

\subsection{Normal aortic valve structure and cell type}

The normal aortic valve consists of three thin and flexible leaflets, which ensures the unidirectional systemic circulation of blood flow from the left ventricle to the aorta during the cardiac cycle. Each leaflet is composed of three layers, i.e., fibrosa, spongiosa and ventricularis (Fig. 1). Fibrosa and ventricularis are both external layers, facing the aorta and the left ventricle, respectively. Fibrosa consists of collagen and VICs that are responsible for reinforcing the valvular structure. The ventricularis consists of collagen and elastin fibers, which provides more compliance and grants the apposition of free edge leaflet regions. Spongiosa is rich in glycosaminoglycans and responsible for absorbing some of the mechanical stress generated during the cardiac cycle. The normal leaflet is avascular and free of inflammatory cells infiltration [15-17].

Normal aortic valve is composed of VECs and VICs. VICs are the major cell population with approximately 5\% myofibroblasts and smooth muscle cells (SMCs) [18]. VECs form a continuous monolayer of cells that are arranged on both sides of the valve, covering the surface of aorta and ventricle. These cells provide the interface between blood and valve, adjacent to the endothelial cell layer of endocardium and adjacent areas of large vessels [19]. As protective barriers for underlying tissues, VECs have shown to inhibit and regulate the pathological proliferation and differentiation of VICs, and maintenance of valve homeostasis through paracrine signals [20]. SMCs only exist at the bottom of ventricle [19]. VICs are the most abundant cells in the aortic valve, distributed in all three layers. Within these layers, the role of extracellular matrix (ECM) is to maintain the proliferation of aortic valve matrix [21]. In healthy adult valves, VECs are mainly of a static fibroblast phenotype and have no synthetic or destructive activity in ECM [19]. The response of VICs to valve injury can be attributed to pathological conditions, such as abnormal hemodynamic or mechanical forces [22]. Evidence also suggests that VICs can also be activated as secretory myofibroblast phenotype [23]. Activated VICs have higher proliferation and motility. VICs specifically express alphasmooth muscle actin protein, which may lead to activated VICs which play a key role in tissue repair and maintaining homeostasis [24].

\subsection{The progression of calcified aortic valve disease}

The progression of CAVD can be divided into three stages: the early stage characterized by VECs damage, the late stage presented with VICs activation, and the end stage indicated with large calcium deposition.

\subsubsection{Early lesions of calcified aortic valve disease}

In the initial stages of CAVD, it is considered that the endothelial damage caused by mechanical stress and shear stress increase. Subsequently, lipids, such as lipoprotein and oxidized low-density lipoprotein cholesterol, penetrate the valve. Then, local subendothelial plaque-like lesions are reported, including inflammatory cells infiltration, lipids accumulation, ECM disorder and inferior elas- 
tic layer rupture [25]. These subendothelial plaque-like lesions represent the earliest pathological changes, preferentially occurring on the lateral surface of the aortic leaflets and extending to the adjacent fibrosa [26]. These pathological findings support the hypothesis that CAVD is an active inflammatory process similar to atherosclerosis [27]. CAVD is similar to atherosclerosis in the initial stages, including early inflammation, macrophage infiltration and foam cell formation. Different cell environment leads to downstream process differentiation after the accumulation of foam cells. CAVD enters the irreversible ossification process through VICs differentiation, resulting in bone formation, valve stenosis and degeneration [26]. Inflammatory cells in the subendothelial and intrafibrous layers produce oxidative stress, a process in which ROS accumulate in cells and cause tissue damage after imbalance of redox system. When the body is exposed to various harmful stimuli can result in oxidative stress, which leads to excessive production of ROS and reactive nitrogen species free radicals. This exceeds the body's ability to scavenge oxides, leading to the imbalance of the oxidative system and antioxidant system, culminating in the functional disorder of tissues or cells $[28,29]$. In addition to oxidative stress, there is release of a variety of growth factors and cytokines, such as transforming growth factor- $\beta$ (TGF- $\beta$ ), tumor necrosis factor- $\alpha$ (TNF- $\alpha$ ), interleukin-1 $\beta$ (IL-1 $\beta)$ and nuclear factor-kappa $\mathrm{B}(\mathrm{NF} \kappa \mathrm{B})$ ligand receptor activator [30-32]. These growth factors and cytokines are the main cause of osteoblast differentiation which occurs through activating a variety of signaling pathways, such as notch, bone morphogenetic protein (BMP), Wnt $/ \beta$-catenin [33] and creating an environment conducive to valve osteogenesis.

\subsubsection{Advanced lesions of calcified aortic valve disease}

With the progress of CAVD, microcalcification can be regarded as small spots of amorphous calcium deposits. These calcified structures are the results of cell death and extracellular vesicles release [34]. Earlier studies have reported that apoptotic bodies of dead cells may provide favorable conditions and starting points for calcification. Stromal vesicles can also be used as nucleation sites for calcium deposition. These observations provide the evidence that numerous matrix vesicles are infiltrated into calcified aortic valves, and participated in the mineralization of natural and biological valves [27]. This kind of microcalcification is usually co-localized in the area of lipoproteins accumulation and inflammatory cells infiltration, which indicates that inflammation plays an initial role in CAVD occurrence [27].

\subsubsection{End stage lesions of calcified aortic valve disease}

When CAVD progresses to the end-stage, calcification extends through the outflow surface of leaflets which is almost transmural and irreversible. Increasing evidence confirmed that valvular calcification in particular is trig- gered by osteoblasts and osteoblast-like phenotypes related molecular pathways [21]. Runt-related transcription factor 2 (RUNX2), osterix and muscle segment homeobox 2 (Msx2) are the initial transcription factors necessary for the identification of osteoblasts. Once the osteoblasts have been differentiated and matured, osteogenic regulatory pathways including notch, Wnt and BMP2 get activated. These cells then secrete and mineralize ECM to deposit calcium [35]. ECM includes type I collagen, alkaline phosphatase, gla protein, proteoglycans, osteopontin and bone sialoprotein. These ECM proteins involved in mineralization are upregulated in calcified valves [36].

\subsection{Pathogenesis of calcific aortic valve disease}

CAVD is a multifactorial disease, affected by environmental and genetic factors, immune molecular pathway, hemodynamic factors and shear stress [15]. The early lesion of CAVD is an active inflammatory process, similar to atherosclerosis, such as lipids deposition, macrophages and T-lymphocytes infiltration, and subsequent endothelial damage [37,38]. However, the end stage of CAVD is often accompanied with heterotopic ossification, including mature lamellar bone formation and active bone reconstruction. At present, many steps in the development of CAVD have been elucidated [39]. Other factors such as the critical transition from inflammatory fibrosis to osteogenesis, or the hypothetical role of microbiota, remain unclear [39].

\subsubsection{Osteoblasts differentiation}

Aortic valve calcification may be related to the osteoblasts differentiation from endothelial cells. CAVD is a closely regulated process similar to bone osteogenesis. Osteogenic differentiation is strictly regulated by a variety of signaling pathways, which is one of the key steps in the progression of valve calcification. Some studies found a specific osteocyte phenotype in calcified valves, which suggests that endothelial cells may have the capacity to differentiate into calcified phenotypes [36]. The possible causes of endothelial cell differentiation include hemodynamic shear stress, ROS, inflammatory cytokines and metabolic syndrome. It is well known that cartilage has the potential to differentiate into calcified phenotypes and has some common characteristics with valves [40]. Chondromodulin-1 is an anti-angiogenic factor that maintains cartilage and heart valves in a vascularized state. Loss of Chondromodulin-1 leads to neovascularization and unusual calcification in the valve matrix. These results suggest that the endochondral ossification pathway may be involved in the progression of aortic valve calcification.

\subsubsection{Lipids}

Aortic valve calcification may also be caused by lipid accumulation, including lipoprotein (a) and its associated oxidized phospholipids [41]. Histological studies revealed lipids accumulation in calcified aortic valve, suggesting that 
lipids could promote the osteogenic differentiation from endothelial cells [42]. As lipids promote chronic inflammatory processes, lipids accumulation may lead to the osteogenic process of aortic valves. It is believed that oxidized lipids mediate valve calcification through the induction of BMP2 [43]. BMP2 exists in aortic valve and upregulates the osteogenic pathway including Msx2, a positive regulator of Wnt pathway. In addition, Wnt expression is elevated in the stenosed aortic valve, subsequently coreceptor formed by low density lipoprotein receptor protein $5 /$ Wnt is activated, which then leads to osteoblasts differentiation. BMP2 also upregulates the osteogenic pathway of cartilage which is involved in RUNX2/core binding factor $\alpha 1$ [44]. Oxidative stress is also associated with inflammation and calcification in surgically resected human aortic valves [45]. ROS mediate the increase of glycogen synthase kinase-3 $\beta$ (GSK-3 $\beta$ ), protein kinase B (AKT), p38 mitogen-activated protein kinase (P38MAPK) expression and signal transduction, which is conducive to osteogenesis [46]. In the previous studies, the relationship between ROS and the rapid progress of CAVD has also been reported [47]. In addition, oxidative stress has been shown to promote calcification [46]. Hence, lipid accumulation and oxidation may induce aortic valve osteogenesis through BMP2 related pathways.

\subsubsection{Inflammation}

Inflammation may be associated with remodeling of calcified aortic valve. There are relatively few macrophages in normal aortic valve, but a large number of leukocytes and macrophages can be found in the calcified valve [48]. Inflammatory factors are secreted by these inflammatory cells, such as IL- $1 \beta$ and $\mathrm{NF} \kappa \mathrm{B}$, promote extracellular matrix remodeling, lipid deposition, fibrosis [49], ossification and calcification [50]. Toll-like receptors are expressed by VICs. They promote osteogenic phenotype of VICs $[51,52]$. NF $\kappa \mathrm{B}$ is activated by TNF$\alpha$ from T-lymphocytes and macrophages [53]. Interleukin6 (IL-6) is known to be secreted by VECs. In vitro studies have shown that IL-6 promotes the mineralization of VECs through BMP2 signaling [14], which plays an important role in the process of aortic valve osteogenesis. In addition, IL-6, a member of NF $\kappa \mathrm{B}$ ligand superfamily, can also induce the expression of receptor activator for $\mathrm{NF} \kappa \mathrm{B}$ ligand (RANKL). It is also observed that RANKL can affect the immune system and control bone regeneration and remodeling [54].

\subsubsection{Angiogenesis and bleeding}

Normal heart valves are avascular and absorb oxygen mainly through blood diffusion. During the onset of the disease, the expression of angiogenic factors leading to neovascularization affects the progress of valvular disease. In other studies, it has been shown that neovascularization occurs in the diseased valve of CAVD patients who undergo surgical AVR [18]. In addition, it has also been observed that the intralobular hemorrhage mainly occurred in the adjacent area of neovascularization, which indicated that the intralobular hemorrhage is caused by these new vessels' rapture [55]. The accumulation of hemoglobin leads to iron overload, oxidative stress, and activates proinflammatory transcription factors. This leads to inflammation and additional angiogenesis. The crosstalk between angiogenesis, oxidative stress and inflammation may play a key role in the progression of CAVD.

\section{Underlying mechanisms of calcified aortic valve disease concomitant with diabetes mellitus}

Previous studies have shown that diabetes can not only accelerate the initiation of CAVD, but also promote the progress of CAVD (Fig. 2) [56]. However, the mechanisms of DM inducing and promoting CAVD remain unclear. Various studies have shown that a series of pathophysiological changes due to diabetes are involved in the pathogenesis of atherosclerosis [57,58]. Studies have also confirmed that CAVD is similar to atherosclerosis in the early pathological process [59], including endothelial damage, lipid deposition, macrophages and T-lymphocytes infiltration [60]. Therefore, we speculate that the early pathogenesis of CAVD complicated with DM may be similar to that of atherosclerosis induced by diabetes. In subsequent sections we review contemporary studies on the pathogenesis of CAVD and DM and the hypothesis of the relationship between these two diseases.

\subsection{Hyperglycemia and advanced glycation end products (AGEs)}

One of the common features of DM is chronic hyperglycemia which has been related to vascular and inflammatory cell interactions with AGEs. AGEs are a heterogeneous group of molecules generated through non-enzymatic glycation and oxidation of proteins such as collagen, elastin, lipids, and nucleic acids which can alter tissue function and its mechanical properties [61]. Hyperglycemia could also increase oxidative stress, destroy protein kinase C (PKC), intracellular signal transduction, and promote inflammation. Thus, hyperglycemia-related AGEs play a deleterious role in cardiovascular diseases. Recent studies have shown that the activation of AGEs can induce endothelialmesenchymal transition and promote the early progression of aortic valve calcification [62].

\subsection{Inflammation}

DM is a kind of chronic and low-grade inflammatory reaction [63]. Numerous clinical studies have shown that DM is often accompanied with upregulation of multiple inflammatory factors [64]. These inflammatory factors mainly include: immune inflammatory response factors, acute reactive proteins, cytokines, and coagulation 


\section{Diabetes Mellitus}

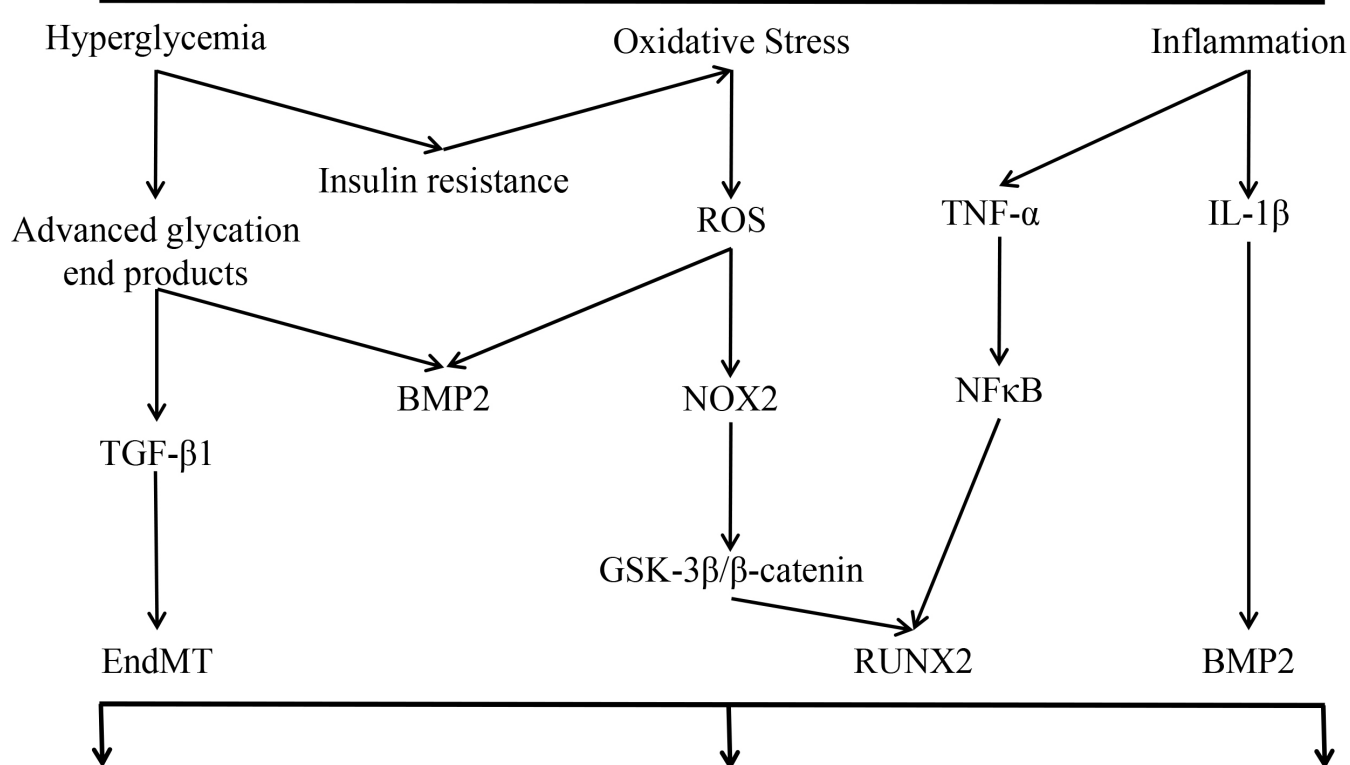

Osteogenic differentiation

Fibroblasts differentiation

Extracellular matrix remodeling

Fig. 2. Underlying mechanisms of calcified aortic valve disease concomitant with diabetes mellitus. ROS, reactive oxygen species; TNF- $\alpha$, tumor necrosis factor $\alpha$; IL- $1 \beta$, interleukin- $\beta$; TGF- $\beta 1$, transforming growth factor- $\beta 1$; BMP2, bone morphogenetic protein 2; NOX2, NADPH oxidase 2; NF $\kappa \mathrm{B}$, nuclear factor kappa B; EndMT, endothelial mesenchymal transformation; GSK-3 $\beta$, glycogen synthase kinase $3 \beta$; RUNX2, runt-related transcription factor 2.

factors [63]. Normal aortic valves consist of relatively few macrophages, while on the other hand, calcified aortic valves consist of a considerably higher number of leukocytes and macrophages. Inflammatory reaction plays an important role in the initiation of CAVD. The inflammatory factors include IL- $1 \beta$ and $\mathrm{NF} \kappa \mathrm{B}$, which promote extracellular matrix remodeling, lipid deposition, fibrosis, ossification and calcification [65]. However, Mosch et al. [4] conducted quantitative histological analysis of aortic valve in patients undergoing AVR, and found CAVD patients convoluted with diabetes may have more advanced disease stage and higher degree of calcification than nondiabetic patients. The early calcification markers were increased in patients with CAVD concomitant with diabetes, but their inflammatory and immune responses were not increased. Therefore, we need to investigate more to explore whether diabetes can induce and aggravate the initiation and progression of CAVD through inflammatory response.

\subsection{Oxidative stress}

ROS mediated oxidative stress plays an important role in the pathophysiology of CAVD. Studies show that overproduction of ROS is associated with hyperglycemia and metabolic disorders [66]. Hyperglycemia and insulin resistance are found in DM patients along with higher levels of various inflammatory factors and growth factors secretion. These factors stimulate the production of oxygen free radicals, increase oxidative stress, and upregulate the level of ROS in calcified aortic valve. Long term exposure to oxidative stress in diabetic patients can induce chronic inflammation and fibrosis in a series of tissues. This promotes the differentiation of valvular mesenchymal cells into myofibroblasts which further differentiate into osteoblasts [47]. ROS can promote aortic valve fibrosis and calcification through NADPH oxidase 2 (NOX2). Specific knockout of NOX2 can significantly inhibit GSK-3 $\beta$ signal and effectively alleviate the progression of CAVD [46]. ROS also mediates the increase of BMP2 expression and its signal transduction, which contributes to osteogenic differentiation and promotes aortic valve calcification [67].

\section{Relation of calcified aortic valve disease and diabetes mellitus}

Aortic valve calcification in diabetic patients may be accomplished through different pathways. In the pathological process of diabetes, there are many metabolic abnormalities, such as hyperinsulinemia, abnormal lipid metabolism, and inflammation [68]. These may also participate in the pathogenesis of CAVD. Diabetes with insulin resistance results in hyperinsulinemia, which is a risk factor for cardiovascular disease [69]. Insulin can also dilate blood vessels. Insulin resistance can weaken the vasodilation effect, it can affect the function of the whole vascular system, and eventually lead to endothelial cells dysfunction [70]. In aortic valve, VECs act as the protective barrier of underlying tissues. These tissues act by regulating per- 
meability, mediating inflammatory cell adhesion, preventing thrombosis, and inhibiting and regulating the pathological proliferation and differentiation of VICs. These cells also maintain the valve homeostasis through paracrine signals. Thus, hyperinsulinemia may induce aortic valve calcification by impairing the function of VECs in diabetic patients. Moreover, Selig et al. [71] have found that hyperinsulinemia combined with hyperglycemia induces impaired AKT- and GSK-3 $\alpha / \beta$-phosphorylation in VICs, which promotes VICs fibrosis. In addition, DM glycosylates lipoprotein and might further trigger formation of immune complexes. LDL as potential antigen can form in situ immune complex in case of severe glycosylation [68]. The immune complexes can stimulate the release of growth factors, cytokines and regulatory factors, which can further accelerate calcification of aortic valves. Growth factors and cytokines are also involved in the pathogenesis of CAVD. In this case diabetes may promote the occurrence and progression of CAVD through the formation of immune complexes. Overall, it is certain that molecules and pathways under CAVD and DM are numerous [71,72]. Many studies have proved that valvular cells cultured with high glucose can increase the expression of TNF- $\alpha$, matrix metalloproteinases, IL- $1 \beta$, extracellular matrix protein. It also activates $\mathrm{PKC}$ signal pathway, BMP signal pathway and TGF- $\beta$ signal pathway, promoting valve remodeling and increasing calcium deposition $[73,74]$. However, additional experimental studies are still needed to confirm and clarify the combined mechanism between the two common diseases.

\section{Future directions}

Confronting the expensive operation cost and poor prognosis of CAVD patients complicated with DM, exploring new medical treatment is crucial. DM induces and aggravates the initiation and progression of CAVD through a variety of pathological processes, such as inflammation and oxidative stress. Inhibition of related pathways by specific drugs may decrease the development and progression of CAVD. Metformin and other drugs may carry out immune regulation through the lipid products of intestinal microbiota, inhibit insulin resistance and hyperglycemia [75], alleviate the chronic inflammatory state of DM and delay the occurrence and progression of CAVD. As a new therapeutic target of CAVD, glucagon-like peptide-1 (GLP-1) down-regulates the expression of RUNX2, Msx2, BMP2 and BMP4, but up-regulates the expression of SRY (sex determining region Y)-box 9 (Sox9) and participates in the mineralization of valve cells [76]. GLP-1 agonist, a new type of DM drug, may become an effective drug intervention to inhibit the progression of CAVD.

\section{Conclusions}

CAVD and DM are both progressive chronic diseases with high morbidity and mortality. Diabetes accelerates the development and progress of aortic valve calcification
[77]. Patients with both of these chronic diseases have a higher risk of adverse events. At present, the effective treatments are surgical AVR or TAVI for CAVD patients. However, diabetic patients have higher risk of thoracotomy, more postoperative complications and worse prognosis, and the implanted bio-artificial aortic valve may degenerate at a younger age [78,79]. The progress of CAVD is an active regulatory process, but the key molecular and signal pathways involved in the process are still unclear, and no effective drug intervention or potential impact on CAVD progress has been identified.

Therefore, in-depth understanding of the pathogenesis of CAVD complicated with DM and the relationship between these two common chronic diseases can help us find appropriate prevention and effective therapy. It will also be beneficial to interrupt the occurrence, delay the progress of calcification, and improve the prognosis of CAVD patients concomitant with diabetes.

\section{Author contributions}

YC and FX designed the study and wrote the manuscript draft. RW supervised the study, guided the structure of the article, and critical revised the draft. All authors contributed to editorial changes in the manuscript. All authors read and approved the final manuscript.

\section{Ethics approval and consent to participate}

Not applicable.

\section{Acknowledgment}

Thanks to all the peer reviewers for their opinions and suggestions.

\section{Funding}

This study was supported by the National Natural Science Foundation of China (No. 81770331 to R.W., and No. 81800340 to F.X.], and Wuxi Health Committee [HB2020012 to F.X.].

\section{Conflict of interest}

The authors declare no conflict of interest.

\section{References}

[1] Bhupathiraju SN, Hu FB. Epidemiology of Obesity and Diabetes and their Cardiovascular Complications. Circulation Research. 2016; 118: 1723-1735.

[2] Otto CM, Prendergast B. Aortic-valve stenosis-from patients at risk to severe valve obstruction. The New England Journal of Medicine. 2014; 371: 744-756.

[3] Myasoedova VA, Ravani AL, Frigerio B, Valerio V, Moschetta $\mathrm{D}$, Songia $\mathrm{P}$, et al. Novel pharmacological targets for calcific aortic valve disease: Prevention and treatments. Pharmacological Research. 2018; 136: 74-82.

[4] Mosch J, Gleissner CA, Body S, Aikawa E. Histopathological assessment of calcification and inflammation of calcific aortic 
valves from patients with and without diabetes mellitus. Histology and Histopathology. 2017; 32: 293-306.

[5] Yan AT, Koh M, Chan KK, Guo H, Alter DA, Austin PC, et al. Association between Cardiovascular Risk Factors and Aortic Stenosis: the CANHEART Aortic Stenosis Study. Journal of the American College of Cardiology. 2017; 69: 1523-1532.

[6] Larsson SC, Wallin A, Håkansson N, Stackelberg O, Bäck M, Wolk A. Type 1 and type 2 diabetes mellitus and incidence of seven cardiovascular diseases. International Journal of Cardiology. 2018; 262: 66-70.

[7] Ljungberg J, Johansson B, Engström KG, Albertsson E, Holmer P, Norberg M, et al. Traditional Cardiovascular Risk Factors and their Relation to Future Surgery for Valvular Heart Disease or Ascending Aortic Disease: A Case-Referent Study. Journal of the American Heart Association. 2017; 6: e005133.

[8] Giritharan S, Cagampang F, Torrens C, Salhiyyah K, Duggan S, Ohri S. Aortic Stenosis Prognostication in Patients with Type 2 Diabetes: Protocol for Testing and Validation of a BiomarkerDerived Scoring System. JMIR Research Protocols. 2019; 8: e13186.

[9] Le Quang K, Bouchareb R, Lachance D, Laplante M, El Husseini D, Boulanger M, et al. Early development of calcific aortic valve disease and left ventricular hypertrophy in a mouse model of combined dyslipidemia and type 2 diabetes mellitus. Arteriosclerosis, Thrombosis, and Vascular Biology. 2014; 34: 2283-2291.

[10] Banovic M, Athithan L, McCann GP. Aortic stenosis and diabetes mellitus: an ominous combination. Diabetes and Vascular Disease Research. 2019; 16: 310-323.

[11] Virani SS, Alonso A, Benjamin EJ, Bittencourt MS, Callaway CW, Carson AP, et al. Heart Disease and Stroke Statistics-2020 Update: A Report From the American Heart Association. Circulation. 2020; 141: e139-e596.

[12] Peeters FECM, Meex SJR, Dweck MR, Aikawa E, Crijns HJGM, Schurgers LJ, et al. Calcific aortic valve stenosis: hard disease in the heart. European Heart Journal. 2018; 39: 2618 2624.

[13] Goody PR, Hosen MR, Christmann D, Niepmann ST, Zietzer A, Adam M, et al. Aortic Valve Stenosis. Arteriosclerosis, Thrombosis, and Vascular Biology. 2020; 40: 885-900.

[14] Akahori H, Tsujino T, Masuyama T, Ishihara M. Mechanisms of aortic stenosis. Journal of Cardiology. 2018; 71: 215-220.

[15] Liu X, Xu Z. Osteogenesis in calcified aortic valve disease: from histopathological observation towards molecular understanding. Progress in Biophysics and Molecular Biology. 2016; 122: 156161.

[16] Wu B, Wang Y, Xiao F, Butcher JT, Yutzey KE, Zhou B. Developmental Mechanisms of Aortic Valve Malformation and Disease. Annual Review of Physiology. 2017; 79: 21-41.

[17] Lauten A, Figulla HR, Möllmann H, Holzhey D, Kötting J, Beckmann A, et al. TAVI for low-flow, low-gradient severe aortic stenosis with preserved or reduced ejection fraction: a subgroup analysis from the German Aortic Valve Registry (GARY). EuroIntervention. 2014; 10: 850-859.

[18] Voisine M, Hervault M, Shen M, Boilard A, Filion B, Rosa M, et al. Age, Sex, and Valve Phenotype Differences in Fibro-Calcific Remodeling of Calcified Aortic Valve. Journal of the American Heart Association. 2020; 9: e015610.

[19] Alushi B, Curini L, Christopher MR, Grubitzch H, Landmesser U, Amedei A, et al. Calcific Aortic Valve Disease-Natural History and Future Therapeutic Strategies. Frontiers in Pharmacology. 2020; 11: 685 .

[20] Hulin A, Hego A, Lancellotti P, Oury C. Advances in Pathophysiology of Calcific Aortic Valve Disease Propose Novel Molecular Therapeutic Targets. Frontiers in Cardiovascular Medicine. 2018; 5: 21.
[21] Rutkovskiy A, Malashicheva A, Sullivan G, Bogdanova M, Kostareva A, Stensløkken K, et al. Valve Interstitial Cells: The Key to Understanding the Pathophysiology of Heart Valve Calcification. Journal of the American Heart Association. 2017; 6: e006339.

[22] Li C, Xu S, Gotlieb AI. The progression of calcific aortic valve disease through injury, cell dysfunction, and disruptive biologic and physical force feedback loops. Cardiovascular Pathology. 2013; 22: 1-8.

[23] Carracedo M, Persson O, Saliba-Gustafsson P, Artiach G, Ehrenborg E, Eriksson P, et al. Upregulated Autophagy in Calcific Aortic Valve Stenosis Confers Protection of Valvular Interstitial Cells. International Journal of Molecular Sciences. 2019; 20: 1486.

[24] Rajamannan NM. Calcific aortic valve disease: cellular origins of valve calcification. Arteriosclerosis, Thrombosis, and Vascular Biology. 2011; 31: 2777-2778.

[25] Clavel M, Messika-Zeitoun D, Pibarot P, Aggarwal SR, Malouf $\mathrm{J}$, Araoz PA, et al. The complex nature of discordant severe calcified aortic valve disease grading: new insights from combined Doppler echocardiographic and computed tomographic study. Journal of the American College of Cardiology. 2013; 62: 2329 2338.

[26] Ohukainen P, Ruskoaho H, Rysa J. Cellular Mechanisms of Valvular Thickening in Early and Intermediate Calcific Aortic Valve Disease. Current Cardiology Reviews. 2018; 14: 264 271.

[27] Kostyunin A, Mukhamadiyarov R, Glushkova T, Bogdanov L, Shishkova D, Osyaev N, et al. Ultrastructural Pathology of Atherosclerosis, Calcific Aortic Valve Disease, and Bioprosthetic Heart Valve Degeneration: Commonalities and Differences. International Journal of Molecular Sciences. 2020; 21: 7434.

[28] Sies H. Oxidative stress: a concept in redox biology and medicine. Redox Biology. 2015; 4: 180-183.

[29] Bhatti JS, Bhatti GK, Reddy PH. Mitochondrial dysfunction and oxidative stress in metabolic disorders - A step towards mitochondria based therapeutic strategies. Biochimica et biophysica Acta. Molecular Basis of Disease. 2017; 1863: 1066-1077.

[30] New SEP, Aikawa E. Molecular Imaging Insights into Early Inflammatory Stages of Arterial and Aortic Valve Calcification. Circulation Research. 2011; 108: 1381-1391.

[31] Lai C, Shao J, Behrmann A, Krchma K, Cheng S, Towler DA. TNFR1-activated reactive oxidative species signals upregulate osteogenic Msx2 programs in aortic myofibroblasts. Endocrinology. 2012; 153: 3897-3910.

[32] Aikawa E, Nahrendorf M, Figueiredo J, Swirski FK, Shtatland $\mathrm{T}$, Kohler RH, et al. Osteogenesis associates with inflammation in early-stage atherosclerosis evaluated by molecular imaging in vivo. Circulation. 2007; 116: 2841-2850.

[33] Hadji F, Boulanger M, Guay S, Gaudreault N, Amellah S, Mkannez $\mathrm{G}$, et al. Altered DNA Methylation of Long Noncoding RNA H19 in Calcific Aortic Valve Disease Promotes Mineralization by Silencing NOTCH1. Circulation. 2016; 134: 1848-1862.

[34] Krohn JB, Hutcheson JD, Martínez-Martínez E, Aikawa E. Extracellular vesicles in cardiovascular calcification: expanding current paradigms. The Journal of Physiology. 2016; 594: 28952903.

[35] Leopold JA. Cellular mechanisms of aortic valve calcification. Circulation. Cardiovascular Interventions. 2012; 5: 605-614.

[36] van Engeland NCA, Bertazzo S, Sarathchandra P, McCormack A, Bouten CVC, Yacoub MH, et al. Aortic calcified particles modulate valvular endothelial and interstitial cells. Cardiovascular Pathology. 2017; 28: 36-45.

[37] Yip CYY, Chen J, Zhao R, Simmons CA. Calcification by valve interstitial cells is regulated by the stiffness of the extracellu- 
lar matrix. Arteriosclerosis, Thrombosis, and Vascular Biology. 2009; 29: 936-942.

[38] Fong F, Xian J, Demer LL, Tintut Y. Serotonin receptor type $2 \mathrm{~B}$ activation augments TNF- $\alpha$-induced matrix mineralization in murine valvular interstitial cells. Journal of Cellular Biochemistry. $2021 ; 122: 249-258$.

[39] Dutta P, Lincoln J. Calcific Aortic Valve Disease: a Developmental Biology Perspective. Current Cardiology Reports. 2018; 20: 21 .

[40] Song R, Fullerton DA, Ao L, Zhao KS, Meng X. An epigenetic regulatory loop controls pro-osteogenic activation by TGF-beta1 or bone morphogenetic protein 2 in human aortic valve interstitial cells. Journal of Biological Chemistry. 2017; 292: $8657-$ 8666.

[41] Tsimikas S. Potential Causality and Emerging Medical Therapies for Lipoprotein(a) and its Associated Oxidized Phospholipids in Calcific Aortic Valve Stenosis. Circulation Research. 2019; 124: 405-415.

[42] Nielsen LB, Grønholdt MLM, Schroeder TV, Stender S, Nordestgaard BG. In Vivo Transfer of Lipoprotein(a) into Human Atherosclerotic Carotid Arterial Intima. Arteriosclerosis, Thrombosis, and Vascular Biology. 1997; 17: 905-911.

[43] Zhang Y, Ma L. Identification of key genes and pathways in calcific aortic valve disease by bioinformatics analysis. Journal of Thoracic Disease. 2019; 11: 5417-5426.

[44] Goto S, Rogers MA, Blaser MC, Higashi H, Lee LH, Schlotter F, et al. Standardization of Human Calcific Aortic Valve Disease in vitro Modeling Reveals Passage-Dependent Calcification. Frontiers in Cardiovascular Medicine. 2019; 6: 49.

[45] Miller JD, Chu Y, Brooks RM, Richenbacher WE, Peña-Silva R, Heistad DD. Dysregulation of antioxidant mechanisms contributes to increased oxidative stress in calcific aortic valvular stenosis in humans. Journal of the American College of Cardiology. 2008; 52: 843-850.

[46] Greenberg HZE, Zhao G, Shah AM, Zhang M. Role of oxidative stress in calcific aortic valve disease and its therapeutic implications. Cardiovascular Research. 2021. (in press)

[47] Liu H, Wang L, Pan Y, Wang X, Ding Y, Zhou C, et al. Celastrol Alleviates Aortic Valve Calcification via Inhibition of NADPH Oxidase 2 in Valvular Interstitial Cells. JACC: Basic to Translational Science. 2020; 5: 35-49.

[48] Raddatz MA, Madhur MS, Merryman WD. Adaptive immune cells in calcific aortic valve disease. American Journal of Physiology-Heart and Circulatory Physiology. 2019; 317: H141-H155.

[49] Liu RM, Gaston Pravia KA. Oxidative stress and glutathione in TGF-beta-mediated fibrogenesis. Free Radical Biology and Medicine. 2010; 48: 1-15.

[50] Cho KI, Sakuma I, Sohn IS, Jo S, Koh KK. Inflammatory and metabolic mechanisms underlying the calcific aortic valve disease. Atherosclerosis. 2018; 277: 60-65.

[51] Zeng Q, Jin C, Ao L, Cleveland JC, Song R, Xu D, et al. Cross-talk between the Toll-like receptor 4 and Notch1 pathways augments the inflammatory response in the interstitial cells of stenotic human aortic valves. Circulation. 2012; 126: S222S230.

[52] Karadimou G, Plunde O, Pawelzik SC, Carracedo M, Eriksson P, Franco-Cereceda A, et al. TLR7 Expression Is Associated with M2 Macrophage Subset in Calcific Aortic Valve Stenosis. Cells. 2020; 9: 1710.

[53] Galeone A, Paparella D, Colucci S, Grano M, Brunetti G. The role of TNF- $\alpha$ and TNF superfamily members in the pathogenesis of calcific aortic valvular disease. TheScientificWorldJournal. 2013; 2013: 875363.

[54] Li X, Lim J, Lu J, Pedego TM, Demer L, Tintut Y. Protective Role of Smad6 in Inflammation-Induced Valvular Cell Calci- fication. Journal of Cellular Biochemistry. 2015; 116: 2354 2364.

[55] Lee A, Wei S, Schwertani A. A Notch more: Molecular players in bicuspid aortic valve disease. Journal of Molecular and Cellular Cardiology. 2019; 134: 62-68.

[56] Scatena M, Jackson MF, Speer MY, Leaf EM, Wallingford MC, Giachelli CM. Increased Calcific Aortic Valve Disease in response to a diabetogenic, procalcific diet in the $\mathrm{LDLr}^{-/-} \mathrm{ApoB}$ 100/100 mouse model. Cardiovascular Pathology. 2018; 34: 28 37.

[57] Stabley JN, Towler DA. Arterial Calcification in Diabetes Mellitus: Preclinical Models and Translational Implications. Arteriosclerosis, Thrombosis, and Vascular Biology. 2017; 37: 205217.

[58] DeFronzo RA. Insulin resistance, lipotoxicity, type 2 diabetes and atherosclerosis: the missing links. the Claude Bernard Lecture 2009. Diabetologia. 2010; 53: 1270-1287.

[59] de Oliveira Sá MPB, Cavalcanti LRP, Perazzo AM, Gomes RAF, Clavel M, Pibarot P, et al. Calcific Aortic Valve Stenosis and Atherosclerotic Calcification. Current Atherosclerosis Reports. 2020; 22: 2 .

[60] Das SR, Everett BM, Birtcher KK, Brown JM, Cefalu WT, Januzzi JL, et al. 2018 ACC Expert Consensus Decision Pathway on Novel Therapies for Cardiovascular Risk Reduction in Patients with Type 2 Diabetes and Atherosclerotic Cardiovascular Disease: a Report of the American College of Cardiology Task Force on Expert Consensus Decision Pathways. Journal of the American College of Cardiology. 2018; 72: 3200-3223.

[61] Yamagishi S, Matsui T. Soluble form of a receptor for advanced glycation end products (sRAGE) as a biomarker. Frontiers in Bioscience (Elite Edition). 2010; 2: 1184-1195.

[62] Kay AM, Simpson CL, Stewart JA. The Role of AGE/RAGE Signaling in Diabetes-Mediated Vascular Calcification. Journal of Diabetes Research. 2016; 2016: 6809703.

[63] Halim M, Halim A. The effects of inflammation, aging and oxidative stress on the pathogenesis of diabetes mellitus (type 2 diabetes). Diabetes \& Metabolic Syndrome: Clinical Research \& Reviews. 2019; 13: 1165-1172.

[64] Signorelli SS, Katsiki N. Oxidative Stress and Inflammation: their Role in the Pathogenesis of Peripheral Artery Disease with or without Type 2 Diabetes Mellitus. Current Vascular Pharmacology. 2018; 16: 547-554.

[65] Deng G, Zhang L, Wang C, Wang S, Xu J, Dong J, et al. AGEsRAGE axis causes endothelial-to-mesenchymal transition in early calcific aortic valve disease via TGF-beta1 and BMPR2 signaling. Experimental Gerontology. 2020; 141: 111088.

[66] Kayama Y, Raaz U, Jagger A, Adam M, Schellinger IN, Sakamoto M, et al. Diabetic Cardiovascular Disease Induced by Oxidative Stress. International Journal of Molecular Sciences. 2015; 16: 25234-25263.

[67] Kim K, Kim D, Lee D, Kim J, Koh J, Kim E, et al. Peroxiredoxin II negatively regulates BMP2-induced osteoblast differentiation and bone formation via PP2a C $\alpha$-mediated Smad1/5/9 dephosphorylation. Experimental \& Molecular Medicine. 2019; 51: 1-11.

[68] Kozakova M, Morizzo C, Goncalves I, Natali A, Nilsson J, Palombo C. Cardiovascular organ damage in type 2 diabetes mellitus: the role of lipids and inflammation. Cardiovascular Diabetology. 2019; 18: 61 .

[69] Balakumar P, Maung-U K, Jagadeesh G. Prevalence and prevention of cardiovascular disease and diabetes mellitus. Pharmacological Research. 2016; 113: 600-609.

[70] Kaur R, Kaur M, Singh J. Endothelial dysfunction and platelet hyperactivity in type 2 diabetes mellitus: molecular insights and therapeutic strategies. Cardiovascular Diabetology. 2018; 17: 121. 
[71] Selig JI, Ouwens DM, Raschke S, Thoresen GH, Fischer JW, Lichtenberg A, et al. Impact of hyperinsulinemia and hyperglycemia on valvular interstitial cells - a link between aortic heart valve degeneration and type 2 diabetes. Biochimica et Biophysica Acta (BBA) - Molecular Basis of Disease. 2019; 1865: 2526-2537.

[72] Selig JI, Boulgaropoulos J, Niazy N, Ouwens DM, Preuss K, Horn P, et al. Crosstalk of Diabetic Conditions with Static Versus Dynamic Flow Environment-Impact on Aortic Valve Remodeling. International Journal of Molecular Sciences. 2021; 22: 6976.

[73] Ciortan L, Macarie RD, Cecoltan S, Vadana M, Tucureanu MM, Mihaila AC, et al. Chronic High Glucose Concentration Induces Inflammatory and Remodeling Changes in Valvular Endothelial Cells and Valvular Interstitial Cells in a Gelatin Methacrylate 3D Model of the Human Aortic Valve. Polymers. 2020; 12: 2786.

[74] Vadana M, Cecoltan S, Ciortan L, Macarie RD, Tucureanu MM, Mihaila AC, et al. Molecular mechanisms involved in high glucose-induced valve calcification in a 3D valve model with human valvular cells. Journal of Cellular and Molecular Medicine. 2020; 24: 6350-6361.

[75] Salazar J, Angarita L, Morillo V, Navarro C, Martínez MS, Chacín M, et al. Microbiota and Diabetes Mellitus: Role of Lipid Mediators. Nutrients. 2020; 12: 3039.
[76] Xiao F, Zha Q, Zhang Q, Wu Q, Chen Z, Yang Y, et al. Decreased Glucagon-Like Peptide-1 Is Associated With Calcific Aortic Valve Disease: GLP-1 Suppresses the Calcification of Aortic Valve Interstitial Cells. Frontiers in Cardiovascular Medicine. 2021; 8: 709741.

[77] Tucureanu MM, Filippi A, Alexandru N, Ana Constantinescu C, Ciortan L, Macarie R, et al. Diabetes-induced early molecular and functional changes in aortic heart valves in a murine model of atherosclerosis. Diabetes and Vascular Disease Research. 2019; 16: 562-576.

[78] Nakamura T, Toda K, Kuratani T, Miyagawa S, Yoshikawa Y, Fukushima S, et al. Diabetes Mellitus Impairs Left Ventricular Mass Regression after Surgical or Transcatheter Aortic Valve Replacement for Severe Aortic Stenosis. Heart, Lung \& Circulation. 2016; 25: 68-74.

[79] Falcão-Pires I, Hamdani N, Borbély A, Gavina C, Schalkwijk CG, van der Velden J, et al. Diabetes Mellitus Worsens Diastolic Left Ventricular Dysfunction in Aortic Stenosis through Altered Myocardial Structure and Cardiomyocyte Stiffness. Circulation. 2011; 124: 1151-1159. 